\title{
Capacitance behaviors of Polyaniline/Graphene Nanosheet Composites Prepared by Aniline Chemical Polymerization
}

\author{
Jieun Kim ${ }^{1}$, Soo-Jin Park ${ }^{2}$ and Seok Kim ${ }^{1, \text {, }}$ \\ ${ }^{1}$ Department of Chemical and Biochemical Engineering, Pusan National University, Busan 609-735, Korea \\ ${ }^{2}$ Department of Chemistry, Inha University, Incheon 402-751, Korea
}

\section{Article Info}

Received 5 October 2012

Accepted 2 January 2013

*Corresponding Author

E-mail: seokkim@pusan.ac.kr

Tel: $+82-51-510-3874$

\section{Open Access}

DOI: http://dx.doi.org/

10.5714/CL.2012.14.1.051

This is an Open Access article distributed under the terms of the Creative Commons Attribution Non-Commercial License (http://creativecommons.org/licenses/ by-nc/3.0/) which permits unrestricted non-commercial use, distribution, and reproduction in any medium, provided the original work is properly cited.

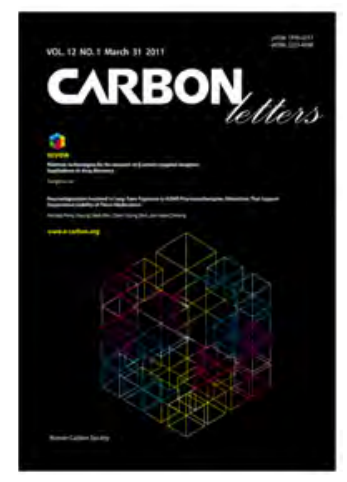

http://carbonlett.org

pISSN: 1976-4251

elSSN: 2233-4998

Copyright $\odot$ Korean Carbon Society

\begin{abstract}
In this study, polyaniline (PANI)/graphene nanosheet (GNS) composites were synthesized through chemical oxidation polymerization by changing the weight ratio of aniline monomers. To examine the morphological structure of the composites, scanning electron microscopy and transmission electron microscopy (TEM) were conducted. TEM results revealed that fibril-like PANI with a diameter of $50 \mathrm{~nm}$ was homogeneously coated on the surface of the GNS. The electrochemical properties of the composites were studied by cyclic voltammetry in $1 \mathrm{M} \mathrm{H}_{2} \mathrm{SO}_{4}$ electrolyte. Among the prepared samples, the PANI/GNS (having $40 \mathrm{wt} \%$ aniline content) showed the highest specific capacitance, $528 \mathrm{Fg}^{-1}$, at $10 \mathrm{mVs}^{-1}$. The improved performance was attributed to the GNS, which provides a large number of active sites and good electrical conductivity. The resulting composites are promising electrode materials for high capacitative supercapacitors.
\end{abstract}

Key words: capacitance behaviors, polyaniline, graphene, chemical polymerization

\section{Introduction}

Graphene, a carbon material with a two-dimensional nanostructure, has attracted a great deal of attention because of its high surface area, electrical conductivity, optimal mechanical stiffness, and high chemical stability [1-3]. In electrochemical measurements, multilayer graphene (from two to ten or more) is considered to be the promising electrode material for supercapacitors [4]. Conducting polymers are among the most extensively studied materials for supercapacitor electrodes, along with carbon and metal oxides. Among the conducting polymers, polyaniline (PANI) is widely used due to its low monomer cost, ease of synthesis, and environmental stability $[5,6]$. However, PANI has several disadvantages such as low cycle life and poor stability, because the oxidation state of emeraldine salt form of PANI can be changed during the repetitive charge/discharge process [7]. The formation of composites of PANI and graphene can potentially be used to reinforce the stability of PANI and to maximize the capacitance value by synergistically exploiting the pseudo-capacitance of PANI and the high conducting and mechanical properties of graphene $[8,9]$.

In this work, composites of PANI/graphene nanosheet (GNS) with different weight ratios are synthesized by chemical oxidation polymerization of aniline monomer (ANI) in an aqueous dispersion of graphene. The combination of the GNS with PANI highly enhanced the electrochemical performance because the GNS serves as a supporting material and provides a large number of active sites. The electrochemical performance, morphology, and chemical structure of the prepared composites have been investigated in this study. 


\section{Experimental}

Graphite oxide was synthesized from natural graphite (SP-1, Bay carbon) by a modified Hummer's method [10]. The graphite powder was added into a mixture of sulfuric acid, sodium nitrate, and potassium permanganate for acid treatment and the solution was maintained at $45^{\circ} \mathrm{C}$ for $2 \mathrm{~h}$. A $30 \% \mathrm{H}_{2} \mathrm{O}_{2}$ aqueous solution was then slowly added into the solution. The oxidized and treated solution was filtered and washed with $\mathrm{HCl}(10 \%)$ and washed with water and ethanol through centrifugation (3600 $\mathrm{rpm}, 5 \mathrm{~min}$ ) to remove residual graphite. GNS was prepared by the reduction of graphene oxide with sodium borohydride $\left(\mathrm{NaBH}_{4}\right)$, as described elsewhere [11]. PANI/GNS composites were synthesized by chemical oxidation polymerization of ANI in the presence of grapheme suspension. A specified amount of GNS was added into $150 \mathrm{ml}$ of $1 \mathrm{M} \mathrm{HCl}$ solution. The solution was sonicated for $2 \mathrm{~h}$. Different amounts of ANI (20, 40, 60 and $80 \mathrm{wt} \%$ ) were then added to the above solution and the solution was sonicated for $30 \mathrm{~min}$. A solution of ammonium persulfate as an oxidizing agent was added dropwise into the above solution. The temperature of the solution was kept at $0-5^{\circ} \mathrm{C}$ during the polymerization reaction. After $24 \mathrm{~h}$, the reaction mixture was filtered and washed with distilled water and ethanol. Finally, it was dried in a vacuum oven at $60^{\circ} \mathrm{C}$. All electrochemical tests were done in a three electrode system. The working electrode was prepared by casting a nafion-impregnated sample onto a glassy carbon electrode. Platinum wire and a saturated calomel electrode were then used as counter and reference electrodes, respectively. The measurements were carried out in $1 \mathrm{M} \mathrm{H}_{2} \mathrm{SO}_{4}$ electrolyte. Electrochemical measurements were performed in an Iviumstat (Ivium Technologies, The Netherlands).

\section{Results and Discussion}

The specific capacitances of the prepared composites with different weight ratios of ANI are presented in Fig. 1. With the introduction of GNS into PANI, the PANI/GNS composites showed improved capacitance compared to that of PANI. In particular, the PANI/GNS composite having $40 \mathrm{wt} \%$ aniline content showed the highest capacitance among the prepared samples and it presented a maximum specific capacitance of $528 \mathrm{Fg}^{-1}$ at $10 \mathrm{mVs}^{-1}$. Fig. 2 shows the cyclic voltammograms of the PANI/GNS composites as a function of aniline content. They show mixed behavior of electric double layer capacitance by the GNS component and redox (or faradaic) capacitance by the PANI. These enhanced specific capacitances are due to the GNS, which provided a large number of active sites and high conductivity [12]. Beyond $60 \mathrm{wt} \%$ aniline content, the composite showed slightly decreased capacitance values. It is thought that aniline content over $60 \mathrm{wt} \%$ would produce an excessively thick coating of PANI onto GNS. It was expected that such a thick PANI coating could not endow effective surface area or a suitable pore structure for easy charge transfer and ion transport. Also, it was observed that the specific capacitance decreases as the scan rates were increased from 10 to $100 \mathrm{mVs}^{-1}$. At a high scan rate, the diffusion of electrolyte ions was limited by structural properties and only the outer active

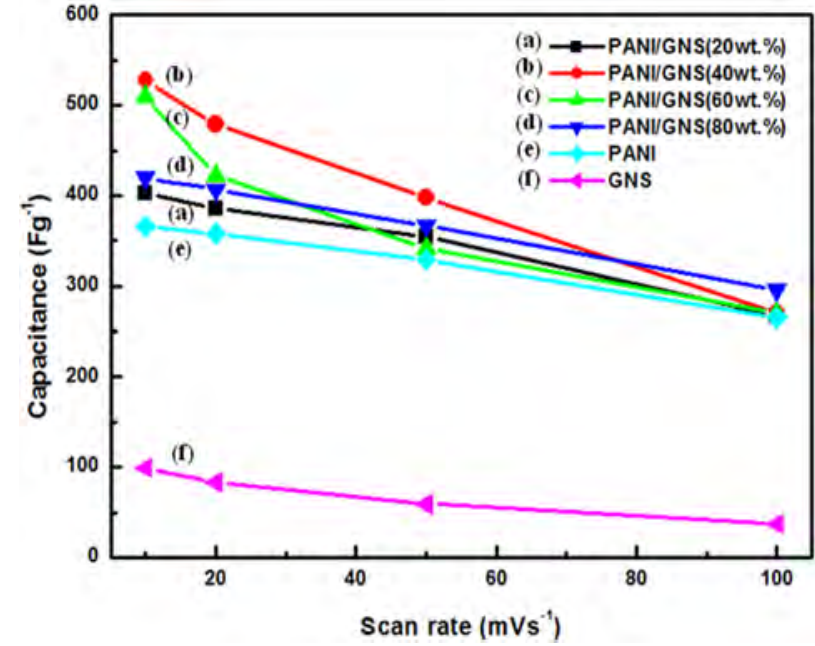

Fig. 1. Specific capacitance of the prepared composites at different scan rates from 10 to $100 \mathrm{mVs}^{-1}$. PANI: polyaniline, GNS: graphene nanosheet.

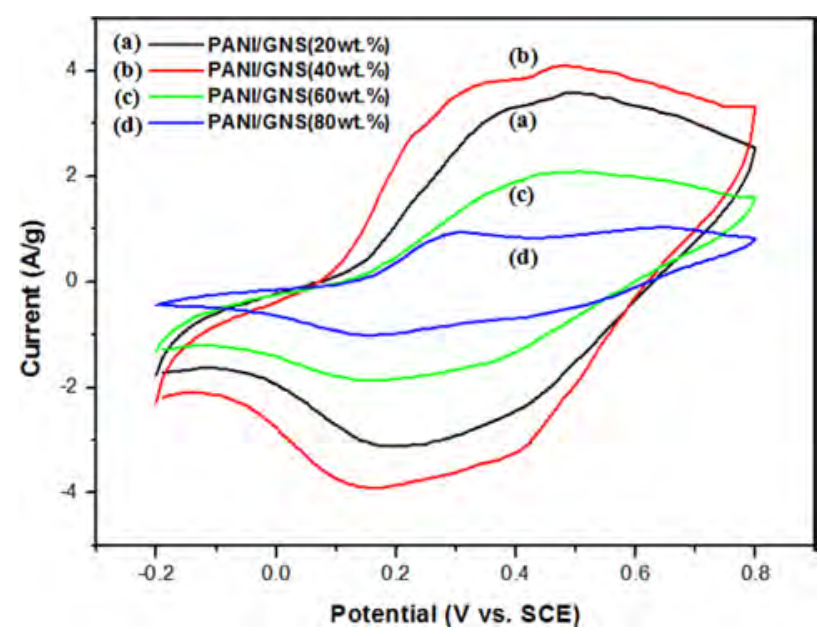

Fig. 2. Cyclic voltammetry curves of the prepared composites with different weight ratios of aniline at a scan rate of $10 \mathrm{mVs}^{-1}$. PANI: polyaniline, GNS: graphene nanosheet.

surface could be utilized for charge storage.

Fig. 3 shows Fourier transform infrared spectroscopy spectra of PANI nanofibers and PANI/GNS (having $40 \mathrm{wt} \%$ aniline content) composites. For PANI nanofibers, the absorption bands at 1563 and $1481 \mathrm{~cm}^{-1}$ are assigned to the $\mathrm{C}=\mathrm{C}$ stretching of quinone rings and benzene rings. The $\mathrm{C}-\mathrm{N}$ stretching vibration of the secondary aromatic amine and aromatic $\mathrm{C}-\mathrm{H}$ bending appeared on the characteristic bands at 1295 and $1110 \mathrm{~cm}^{-1}[13,14]$. Also, these bands are observed in the spectrum of PANI/GNS (having $40 \mathrm{wt} \%$ aniline content) composites. The absorption band at $1563 \mathrm{~cm}^{-1}$ is attributed to the skeletal vibration of the GNS, which overlapped with the $\mathrm{C}=\mathrm{C}$ stretching of quinone rings of PANI [15]. The morphology and structural properties of the composites were examined by scanning electron microscopy and transmission electron microscopy images presented in Fig 4. Transparent and wrinkled graphene sheets can be seen in Fig. 4b. In the PANI/GNS (having $40 \mathrm{wt} \%$ aniline content) composite, the surface of the GNS was coated by fibril-like PANI with 


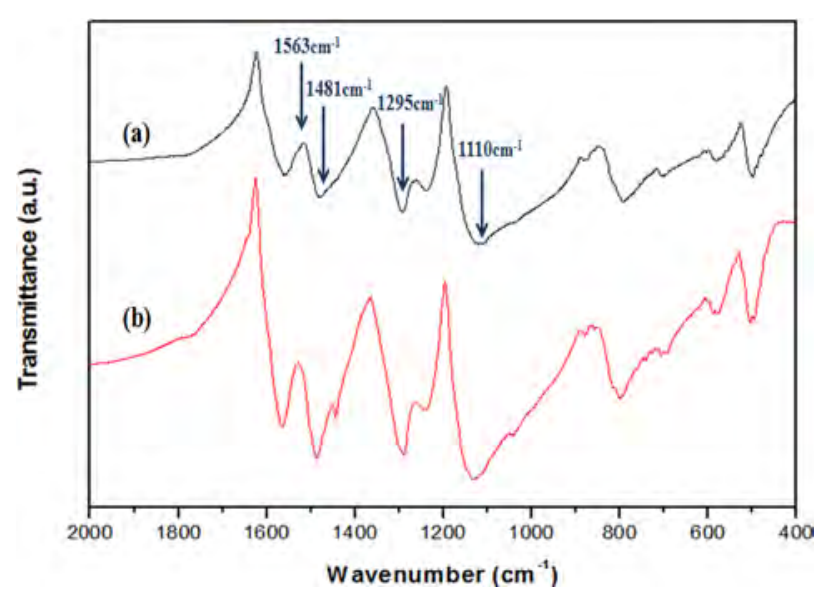

Fig. 3. Fourier transform infrared spectroscopy spectra of (a) polyaniline (PANI) nanofibers and (b) PANI/graphene nanosheet composite (having $40 \mathrm{wt} \%$ aniline content).

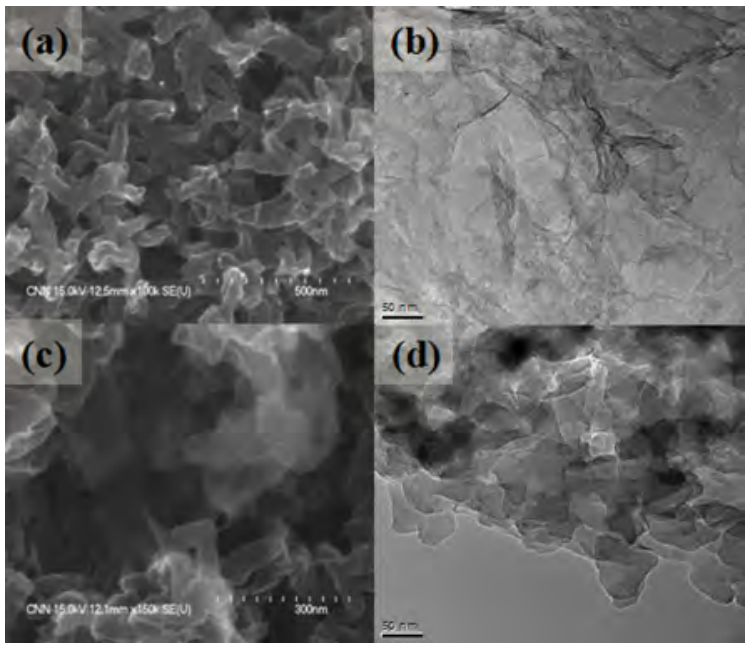

Fig. 4. (a) Scanning electron microscopy (SEM) image of pristine polyaniline (PANI), (b) transmission electron microscopy (TEM) image of graphene nanosheet (GNS) and (c) SEM and (d) TEM images of PANI/GNS (having $40 \mathrm{wt} \%$ aniline content).

$50 \mathrm{~nm}$ average diameter. Due to the electrostatic attraction between PANI and the functional groups of GNS, the PANI nanofibers were coated on the surface of the GNS [16].

\section{Conclusions}

In the present work, PANI/GNS composites were prepared via chemical oxidation polymerization by changing the weight ratio of ANI. The PANI/GNS composite (having $40 \mathrm{wt} \%$ aniline content) shows high specific capacitance of $528 \mathrm{Fg}^{-1}$ at $10 \mathrm{mVs}^{-1}$. The enhanced capacitance of the composite electrodes is attributed to the combined effects of PANI and GNS. The introduction of GNS into PANI increased not only the conductivity but also the number of active sites, which improved the charge transfer and ion transport throughout the electrode. The PANI/GNS composites with high specific capacitance are promising electrode materials for supercapacitors.

\section{Acknowledgments}

This research was partly supported by the Ministry of Knowledge and Economy (Materials Source Technology Project, Grant No. 10037238.2011). It was also partly supported by Inha University through the Korea Institute for Advancement of Technology, Korea (Wide-range Economic Core Institute Project, Preparation and Application of High Performance Artificial Graphite).

\section{References}

[1] Wang X, Bai H, Yao Z, Liu A, Shi G. Electrically conductive and mechanically strong biomimetic chitosan/reduced graphene oxide composite films. J Mater Chem, 20, 9032 (2010). http://dx.doi. org/10.1039/C0JM01852J.

[2] Castro Neto AH, Guinea F, Peres NMR, Novoselov KS, Geim AK. The electronic properties of graphene. Rev Mod Phys, 81, 109 (2009). http://dx.doi.org/10.1103/RevModPhys.81.109.

[3] Park SE, Park SJ, Kim S, Preparation and capacitance behaviors of cobalt oxide/graphene composites, Carbon Lett, 13, 130 (2012). http://dx.doi.org/10.5714/CL.2012.13.2.130.

[4] Zhang LL, Zhou R, Zhao XS. Graphene-based materials as supercapacitor electrodes. J Mater Chem, 20, 5983 (2010). http://dx.doi. org/10.1039/C000417K.

[5] Radoicic M, Saponjic Z, Nedeljkovic J, Ciric-Marjanovic G, Stejskal J. Self-assembled polyaniline nanotubes and nanoribbons/ titanium dioxide nanocomposites. Synth Met, 160, 1325 (2010). http://dx.doi.org/10.1016/j.synthmet.2010.04.010.

[6] Rahy A, Yang DJ. Synthesis of highly conductive polyaniline nanofibers. Mater Lett, 62, 4311 (2008). http://dx.doi.org/http:// dx.doi.org/10.1016/j.matlet.2008.06.057.

[7] Misoon O, Seok K. Effect of dodecyl benzene sulfonic acid on the preparation of polyaniline/activated carbon composites by in situ emulsion polymerization. Electrochim Acta, 59, 196 (2012). http:// dx.doi.org/http://dx.doi.org/10.1016/j.electacta.2011.10.058.

[8] Yan Y, Cheng Q, Wang G, Li C. Growth of polyaniline nanowhiskers on mesoporous carbon for supercapacitor application. J Power Sources, 196, 7835 (2011). http://dx.doi.org/http://dx.doi. org/10.1016/j.jpowsour.2011.03.088.

[9] Park DY, Lim YS, Kim MS, Performance of expanded graphite as anode materials for high power Li-ion secondary batteries, Carbon Lett, 11, 343 (2010). http://dx.doi.org/10.5714/CL.2010.11.4.343.

[10] Hummers WS, Offeman RE. Preparation of graphitic oxide. J Am Chem Soc, 80, 1339 (1958). http://dx.doi.org/10.1021/ ja01539a017.

[11] Liang Y, Wu D, Feng X, Müllen K. Dispersion of graphene sheets in organic solvent supported by ionic interactions. Adv Mater, 21, 1679 (2009). http://dx.doi.org/10.1002/adma.200803160.

[12] Li J, Xie H, Li Y, Liu J, Li Z. Electrochemical properties of graphene nanosheets/polyaniline nanofibers composites as electrode for supercapacitors. J Power Sources, 196, 10775 (2011). http:// dx.doi.org/10.1016/j.jpowsour.2011.08.105.

[13] Li G, Jiang L, Peng H. One-dimensional polyaniline nanostructures with controllable surfaces and diameters using vanadic acid as the oxidant. Macromolecules, 40, 7890 (2007). http://dx.doi. org/10.1021/ma070650o.

[14] Wei Z, Zhang L, Yu M, Yang Y, Wan M. Self-assembling sub- 
micrometer-sized tube junctions and dendrites of conducting polymers. Adv Mater, 15, 1382 (2003). http://dx.doi.org/10.1002/ adma.200305048.

[15] Li Y, Peng H, Li G, Chen K. Synthesis and electrochemical performance of sandwich-like polyaniline/graphene composite nanosheets. Eur Polym J, 48, 1406 (2012). http://dx.doi.org/http:// dx.doi.org/10.1016/j.eurpolymj.2012.05.014.

[16] Yan J, Wei T, Shao B, Fan Z, Qian W, Zhang M, Wei F. Preparation of a graphene nanosheet/polyaniline composite with high specific capacitance. Carbon, 48, 487 (2010). http://dx.doi.org/http:// dx.doi.org/10.1016/j.carbon.2009.09.066 